\title{
Formula Manipulation in the Bond Graph Modelling and Simulation of Large Mechanical Systems
}

\author{
by A. M. BOS and M. J. L. TIERNEGO
}

Department of Electrical Engineering, Twente University of Technology, P.O. Box 217, 7500 AE Enschede, The Netherlands

ABSTRACT: A multibond graph element for a general single moving body is derived. A multibody system can easily be described as an interconnection of these elements. 3-D mechanical systems usually contain dependent inertias having both differential and integral causality. $A$ method is described for the transformation of inertias with differential causality to an integral form, using formula manipulation. The program also helps to find experimentally the optimal choice for the generalized coordinates. The resulting explicit differential equation may be solved using a standard integration routine or simulation program.

\section{Nomenclature}

$\mathbf{x}_{k, l}^{i} \quad$ column matrix with the displacement of point $k$ relative to $l$, in coordinates of system $i$. The subscript $l$ is omitted when $l=0$

$\boldsymbol{\Omega}_{k, l}^{i}$ column matrix with the angular velocities of body $k$, relative to body or coordinate system $l$, in coordinates of coordinate systcm $i$. The subscript $l$ is omitted when $l=0$ (the inertial system)

$\dot{\mathbf{x}}_{p}^{i, j} \quad$ column matrix with the relative velocity of point $p$ in coordinates of system $i$, measured with respect to the coordinate system $j$. When $j=0, j$ is omitted

$\mathbf{A}^{j, i}$ Coordinate transformation matrix from system $i$ to $j$

$\mathbf{X}\left(\mathbf{x}_{p, i}^{i}\right)$ transformation matrix giving the linear velocity of point $p$ due to rotation of system $i$, in coordinates of system $i$

$\mathbf{F}_{p}^{i}$ force acting on the point $p$, in coordinates of base $i$

$\mathbf{M}_{j}^{i}$ moment acting on body $j$, in coordinates of base $i$

$\mathbf{m}_{i}$ diagonal matrix with the mass of body $i$

$\mathbf{J}_{i}^{i}$ inertia matrix of body $i$, relative to the body fixed system $i$

$\mathrm{R}_{i}$ dissipator $i$

$\mathrm{C}_{i} \quad$ compliance $i$

$\dot{\mathbf{q}}_{a}$ column matrix with velocities, in which " $a$ " stands for: $i, I, \mathrm{C}, \mathrm{R}, \mathrm{S}, d$.

$\mathbf{T}_{b, i}$ matrix relating velocities $\dot{\mathbf{q}}_{b}$ to the independent velocities $\dot{\mathbf{q}}_{i}$, in which " $b$ " stands for: $I, \mathrm{C}, \mathrm{R}, \mathrm{S}$.

The underlined characters in the figures are equivalent to the bold face characters, representing a matrix.

\section{Introduction}

A systematic approach is necessary in modelling large mechanical systems. In this paper a description of the kinematics is given first, which forms the basis for the 
junction structure of the mechanical bond graph. A uniform notation of velocities is introduced. With the elements attached to this junction structure the dynamics of the system is described using the power conserving concept of bond graphs.

A bond graph model of a system is a basis for the formulation of equations or for simulation. Constraints in mechanical systems may lead to implicit differential equations. In the second part of the paper it is explained how formula manipulation is used to obtain an explicit differential equation which can be solved with a standard simulation program. An industrial manipulator is used as an example to demonstrate the method.

\section{Modelling Mechanical Systems}

\section{Kinematics}

In the description of mechanical systems, displacements and velocities play an important role. Describing large systems requires a systematic notation of velocities, which will be discussed together with the kinematics. Wittenburg (1) gave a good description of kinematics and dynamics, while Allen (2) introduced a comprehensive notation of velocities. These notations are combined and augmented.

Velocities have a magnitude and direction, represented by a vector. A vector will be described as a linear combination of three orthonormal vectors, called a base system, coordinate system or for short a base [Eq. (1a)]. The three $x, y$ and $z$ base vectors form a righthanded system. Vectors will be denoted with an arrow on top, base vectors are additionally identified with the letter $\mathbf{e}$. The scalars in this equation are the coordinates of the given vector in base 0 . In (1b) these coordinates are represented in one column matrix, i.e.

$$
\begin{aligned}
& \dot{\mathbf{x}}_{p}=\dot{x}_{p}^{0 x} \cdot \overrightarrow{\mathbf{e}}^{0 x}+\dot{x}_{p}^{0 y} \cdot \overrightarrow{\mathbf{e}}^{0 y}+\dot{x}_{p}^{0 z} \cdot \overrightarrow{\mathbf{e}}^{0 z} \\
& \dot{\mathbf{x}}_{p}^{0}=\left[\begin{array}{lll}
\dot{x}^{0 x} & \dot{x}^{0 y} & \dot{x}^{0 z}
\end{array}\right]^{T} .
\end{aligned}
$$

In mechanical systems more coordinate systems are used simultaneously, which are identified by a superscript; so the superscript in (1) denotes that the vector is described in base 0 . The subscript denotes which vector is described. The coordinates of a vector are dependent on the orientation of the base. The coordinates of a vector described in a certain base $i$ are transformed to base $j$ by a coordinate transformation (2). Transformation matrices are denoted by capital A. The orientation of two bases with respect to each other is specified with cardan angles, which also defines the transformation matrices

$$
\dot{\mathbf{x}}_{p}^{j}=\mathbf{A}^{j, i} \cdot \dot{\mathbf{x}}_{p}^{i}
$$

Figure 1 shows an arbitrary moving body with a body-fixed coordinate system, a centre of gravity and a moving point $p$. The body is described with respect to the inertial base 0 . The velocity of point $p$ is in vector notation described with the well known Eq. (3). In (4a) the velocity of $p$ is given in the matrix notation. Equivalent to the cross product in (3) is the matrix product in (4) using the antisymmetric matrix $\mathbf{X}$ with the coordinates of the point $p$ in base 1 as argument (4b). In (4) the subscripts consist of two numbers. The first indicates at which point the velocity is described, 


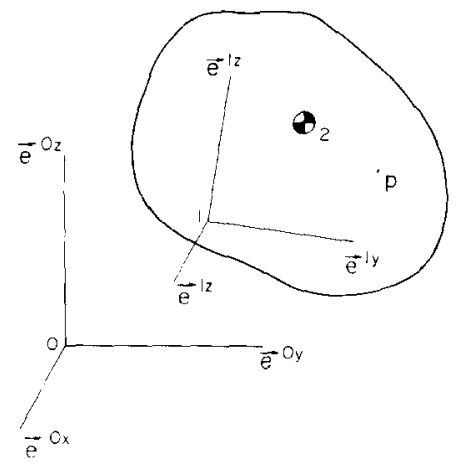

FIG. 1. Arbitrary moving body.

the second relative to which point this velocity is meant. The last term in (4a) is the linear velocity of $p$ due to the rotational velocity $\Omega_{1,0}^{1}$ of base 1 with respect to base 0 . This specific velocity, which appears often in the bond graph, is denoted with an $r$ after the superscript (5), that is

$$
\begin{gathered}
\dot{\mathbf{x}}_{p}=\dot{\mathbf{x}}_{1,0}+\dot{\mathbf{x}}_{p, 1}+\dot{\mathbf{\Omega}}_{1,0}^{1} X \overrightarrow{\mathbf{x}}_{p, 1} \\
\mathbf{X}\left(\mathbf{x}_{p, 1}^{1}\right)=\left[\begin{array}{ccc}
0 & x_{p}^{1 z} & -x_{p}^{1 y} \\
-x_{p}^{1 z} & 0 & x_{p}^{1 x} \\
x_{p}^{1 y} & -x_{p}^{1 x} & 0
\end{array}\right] \\
\dot{\mathbf{x}}_{1,0}^{0}+\mathbf{A}^{0,1} \cdot\left(\dot{\mathbf{x}}_{p, 1}^{1}=\mathbf{X}\left(\mathbf{x}_{p, 1}^{1}\right) \cdot \mathbf{\Omega}_{1,0}^{1}\right) \\
\mathbf{X}\left(\mathbf{x}_{p, 1}^{1}\right) \cdot \mathbf{\Omega}_{1,0}^{1} .
\end{gathered}
$$

The velocity relation in (4) forms the basis for the bond graph description of mechanical systems, which is represented by the bond graph junction structure of Fig. 2 [see Refs. (3) and (4)]. The three components of the velocities are represented by the three components of a multibond. (The expression multibond is preferred over the word vectorbond.) The notation used is according to Breedveld (5). The multiport transformers represent the matrices in (4), as can be seen on the corresponding constitutive matrices. With the described notation only the kinematics of a system can be modelled. Our main interest is in the dynamics.

\section{Dynamics}

To represent the dynamics, the bond graph of Fig. 2 is augmented with inertia elements, and the linear velocity of the centre of gravity is described, so one

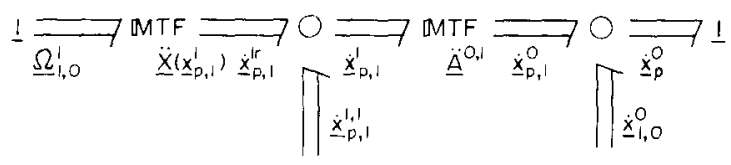

FIG. 2. Junction structure representing kinematics of a free moving body. 


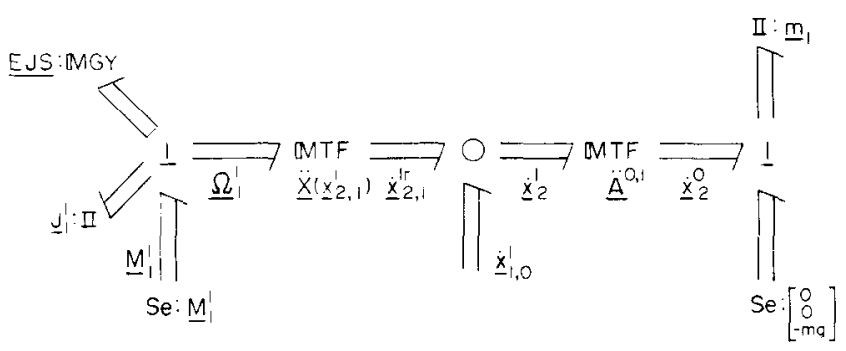

Fig. 3. Dynamics of a moving body.

0-junction disappears (Fig. 3). At the right side the mass matrix $\mathbf{m}$ is connected as an I-element to the 1-junction of the translational velocities in the inertial frame, together with the gravity force source Se, which acts in the vertical direction only. At the left side the diagonal moment of inertia matrix $\mathbf{J}$ is connected as an I-element to the 1-junction of the rotational velocities in the body frame. The gyroscopic forces which act between these velocities are represented by the modulated gyrator MGY. Because the Euler equations represent these forces, the MGY is referred to as an Eulerian junction structure (EJS). Finally, an external moment-source $\mathbf{M}$ is supposed to act on this 1 -junction.

From the bond graph Eq. (6) can be derived. It describes the sum of the efforts (moments) at the left 1-junction of Fig. 4 . The first term represents the moment

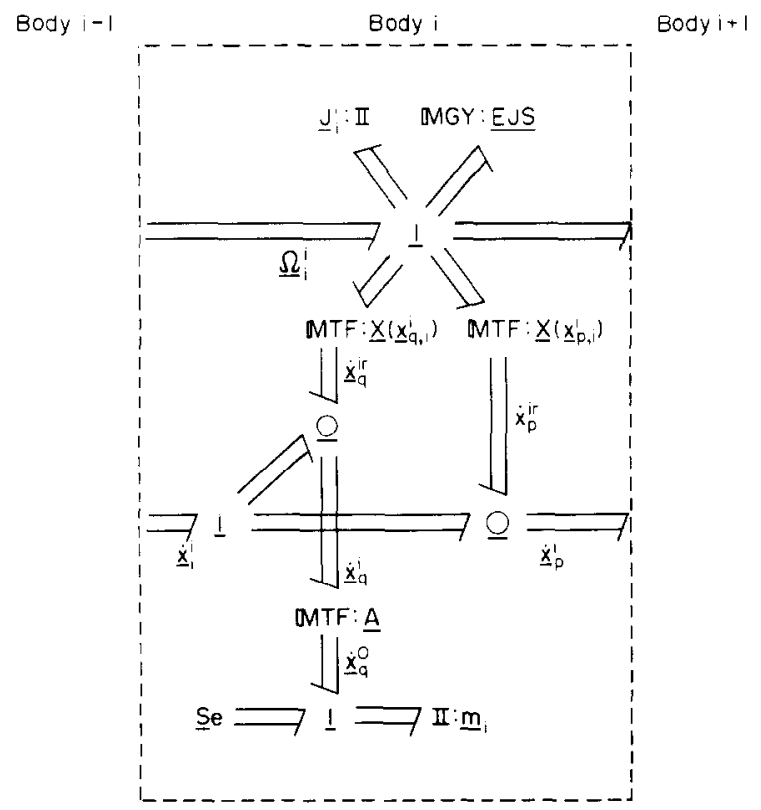

FIG. 4. Bond graph representing the dynamics of a link $i$ in a multibody system, with centre of gravity $q$ and connection point $p$. 
required for the angular accelleration of the inertia of the body. The inertia is described in the centre of gravity with the same orientation as the body fixed coordinate system. The second term represents the modulated gyrator for the coriolis and gyroscopic forces. The last term is the moment required for the translational acceleration of the centre of gravity due to the gravitation and possible other forces acting on this centre of gravity. The first two terms in (6) are equivalent to the Euler equations; the last term is related to the Huygens-Steiner rule. Left of the equal sign in (6) is the external moment source Se, i.e.

$$
\mathbf{M}_{1}^{1}=\mathbf{J}_{1}^{1} \cdot \mathbf{\Omega}_{1}^{1}+\mathbf{\Omega}_{1}^{1} X \mathbf{J}_{1}^{1} \cdot \mathbf{\Omega}_{1}^{1}+\mathbf{X}\left(\mathbf{x}_{2,1}^{1}\right)^{T} \cdot A^{1,0} \cdot\left(\mathbf{m}_{1} \cdot \ddot{\mathbf{x}}_{2}^{0}-\mathbf{F}_{z}^{0}\right)
$$

Using this representation of one moving body, a bond graph of an interconnected multibody system can be derived. In Fig. 4 the bond graph of the $i$ th body is given, which is based on Fig. 3. In this bond graph the upper 1-junction together with the upper horizontal bonds represent the angular velocity of this body, in body coordinates. The lower horizontal bonds represent the translational velocity of body $i$ and of its connection in point $p$ with body $i+1$, also in body coordinates. The vertical branches represent the interaction between rotation and translation as representated in Fig. 3. Two branches occur : the left one representing the interaction with the centre of gravity $q$, the right one is related to a fixed point $p$ in which the translational coupling with a body $i+1$ is supposed to take place. If coupling with another body in another point exists, a third vertical branch has to be added.

During the construction of the bond graph of a large system it is useful to have a graph in which only the overall structure of the system is shown, while the bodies are not worked out in such detail as in Fig. 4. The word bond graph is suited for this purpose. In Fig. 5 the general word bond graph for a part of a multibody system is shown. In this, each body is represented by a block interconnected by two rows of bonds. The upper relates to the angular velocities, the lower to the linear velocities. Each body is described in its body-fixed coordinate system. Therefore, coordinate transformations between the body fixed coordinate systems are required and shown as MTF's. At the 0-junctions the open bonds of the difference velocities at the interconnections can be seen. Drives or constraints can be applied to these bonds.

After the structure of a system in the form of a word bond graph has been drawn,

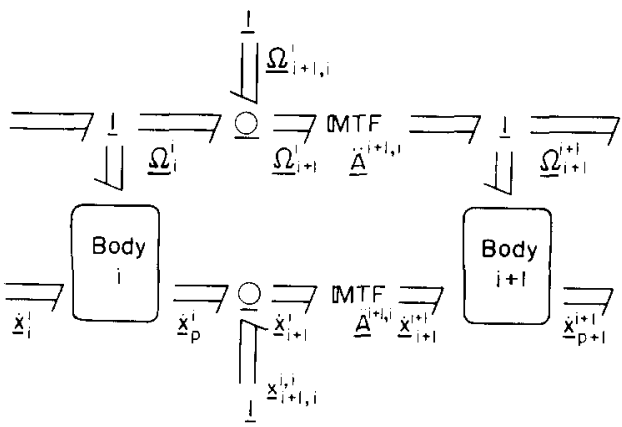

FIG. 5. Word bond graph of a link in a multibody system. 
each part may be separately worked out in detail, if required. The discussed modelling method will next be applied to an industrial manipulator.

\section{Modelling an industrial manipulator}

The mechanism, as shown in Fig. 6, consists of three rigid bodies. For bodies 1 and 2 , body-fixed coordinate systems are chosen respectively as base 1 and base 2 . Body 3 is described in the same coordinate system as body 2 , which can be done when they have the same angular velocity. The actuators in the system are an electric PM motor and two hydraulic motors, the electric motor being used for the angular displacement of body 1 in the $1 z$ direction. The hydraulic motors are used for the angular displacement of bodies 2 and 3 in the $2 x$ direction and for the linear displacement of body 3 with respect to body 2 in the $2 y$ direction. The compliance of the oil in the hydraulic system has been taken into account. The compliances of the links are neglected. When a more accurate model is needed they can casily be added.

A word bond graph is drawn in Fig. 7. Bodies 2 and 3 are described in the same block. The difference in angular velocity between body 1 and 2 is given in the upper row of bonds at the 0 -junction with the drive 2 attached to it. In the same way a moment for the angular velocity of body 1 at the $1 z$ axis is represented with an effort source, drive 1 . The linear velocity between bodies 2 and 3 is applied within the right block, so the point of application of drive 3 is not shown in the word bond graph. The velocity of point 35 has to be known and is therefore an explicit 1 -junction in the word bond graph.

The word bond graph can be worked out in detail to give rise to the bond graph of the industrial manipulator, Fig. 8. Because in this particular case the velocities of points 10 and 2 are zero the branches in the bond graph belonging to body 1 are eliminated. The three remaining branches left belong to bodies 2 and 3 . From left to right they give the velocity of the centre of gravity of bodies 2 and 3 respectively. The last one gives the velocity of the endpoint 35 . The difference velocity between bodies 2 and 3 is added to the relative velocities of points 30 and 35 , both in the $y$-direction. Some elements in this bond graph need explanation. The $\mathrm{C}$-elements represent oil compressibility. The inertias of bodies 2 and 3 are added to one inertia while both bodies have equal angular velocity. The same holds for the corresponding gyrator.

The bond graph modelling method as explained and applied to an industrial manipulator may be used to describe the dynamics of large mechanical systems. In

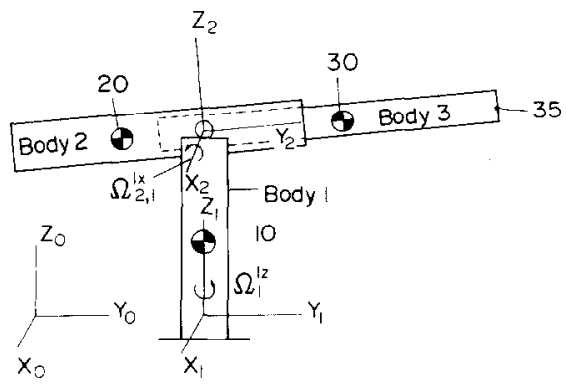

FIG. 6. Industrial manipulator. 


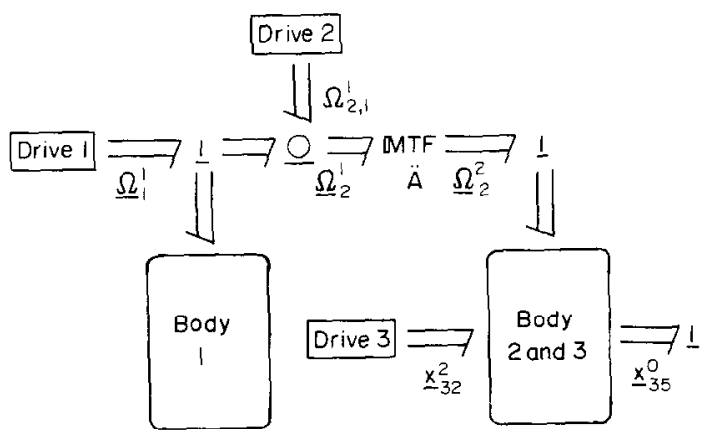

FIG. 7. Word bond graph of the industrial manipulator.

the third part of this paper simulation will be discussed, because mechanical bond graphs have some specific problems, related to the kinematic constraints in these systems.

\section{Simulation}

Before a system, described by a bond graph, can be simulated the output variable of each element and junction has to be known. By adding causal strokes to the bonds

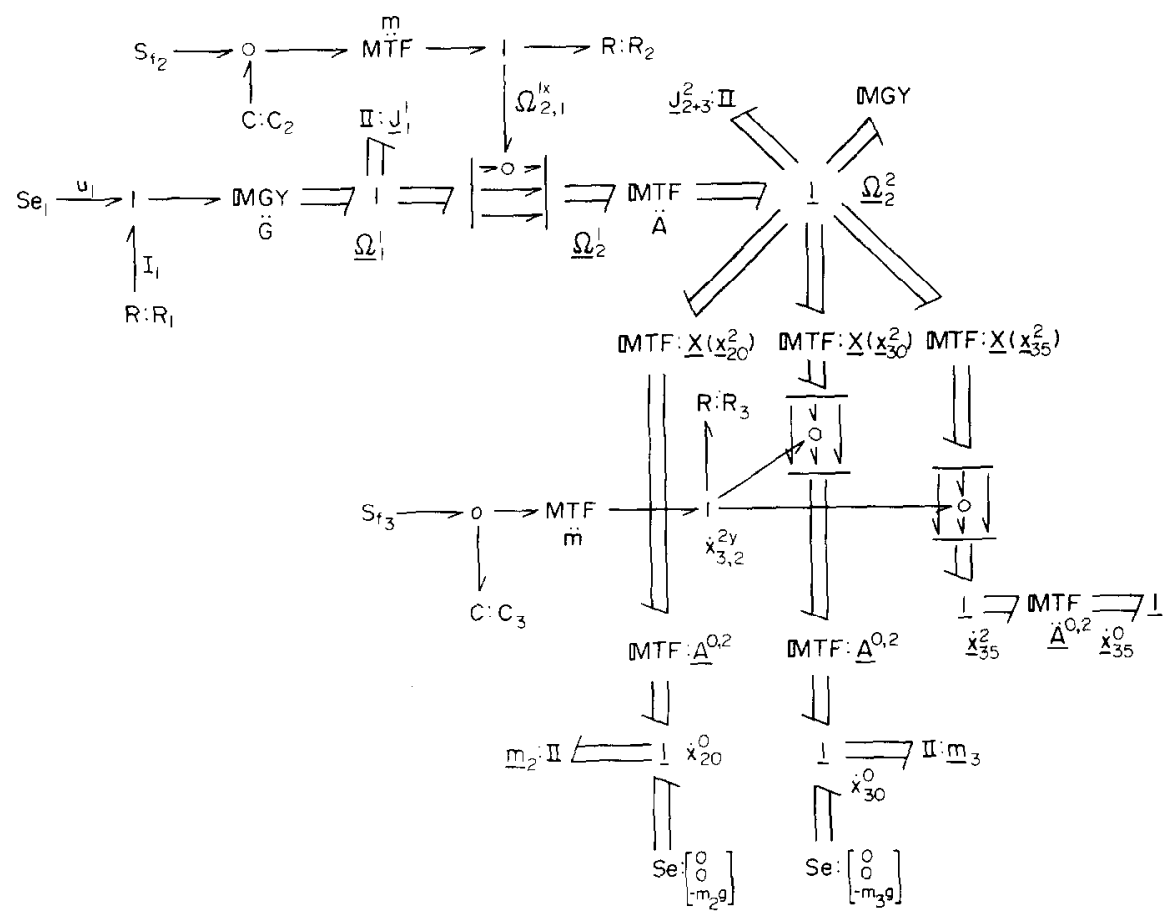

FIG. 8. Bond graph of the industrial manipulator. 
the output variable of each element is shown. Usually in mechanical systems, many inertias will have differential causality, indicating their dependence on inertias in integral causality. The mathematical equivalent is a set of implicit differential equations. This appearance of mixed integral and differential causality for the inertias is due to the many kinematic constraints, common in mechanical systems. In general, this will result in numerical problems during the simulation on a digital computer.

There are several ways to simulate a mechanical system, described by a bond graph, each having its specific disadvantages. First, it is possible to choose integral causality for all storage elements, resulting in a non-causal bond graph, which is mathematically equivalent to an implicit set of differential equations. Simulation is in principle possible using iterative solution methods. A second way is to release the mechanical system by adding damped compliances on the desired places in the bond graph, see Margolis and Karnopp (6). Usually this results in long simulation times due to the introduced stiffnesses. A third way is based on the transformation of the differential inertias to the integral inertias. In contrast to the first method, the second and the third imply that the implicit differential equation is transformed to an explicit form. A disadvantage of the third method is, that it is manually hardly possible to execute for other than small systems. Allen (7) proposed to computerize the method by using formula manipulation for multiplying and inverting matrices. Instead of starting from the bond graph he started however from the displacement equations for the computation of the desired matrices.

In the next section a method is described which starts from the bond graph of a mechanical system. The junction structure of the bond graph is equivalent to the velocity relations of the system. Formula manipulation is used to construct the explicit differential equation from these velocity relations and the element relations.

A general form of the method will be derived using a small example system. An application and some simulation results are shown for an industrial manipulator, already modelled in the first part of this paper.

\section{Derivation of the procedure}

The example to be used (Fig. 9) is a massless rod rotating around the $y$-axis. A mass can move along the rod and is attached to the rod by a linear spring.

The corresponding bond graph, presented in Fig. 10, is composed in the way described. The MGY-element, which in general is connected to the upper 1-junction for the angular velocity of the body together with the inertia, is left out : it will have

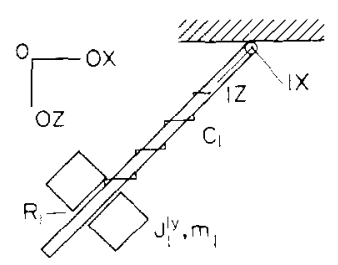

FIG. 9. Example system. 


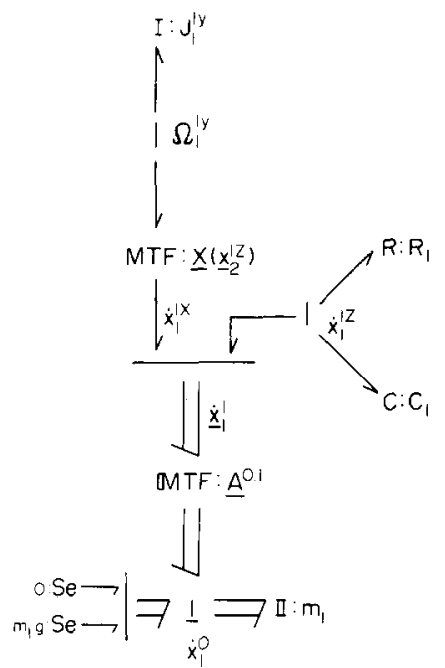

FiG. 10. Bond graph of the example system.

no effect as long as there is a rotation in only one direction. Adding the causal strokes shows that one of the inertial elements gets differential causality and is dependent on the others. Its computation by numerical differentiation causes instability if the corresponding loop gain is larger than onc (8). In this casc, a loop gain smaller than one can be obtained, but in general in larger systems, in which the loop gains may even be state dependent, this no longer possible.

A better way to solve the problem is to transform the dependent inertias to the others. For small mechanical systems like the one presented this can easily be done by hand. For larger systems, however, a manual procedure becomes very difficult. In the following it will be shown how such a procedure can be computerized.

It is preferable to restrict our procedure to that part of the bond graph in which all dependencies occur. Allen (2) called such a part with many kinematic constraints a "mechanism". In our example, the complete bond graph has to be treated.

In the considered (sub)bond graph all inertias will be represented as one multiport I-elemerit connected to a junction structurc. Afterwards other clements will bc treated in the same way.

The junction structure represents all the velocity relations of the system. These velocity relations (7), which can immediately be obtained from the original bond graph, are a set of linear equations with non-constant coefficients :

$$
\left.\begin{array}{rl}
x_{1}^{1 z} \cdot \Omega_{1}^{1 y} & =\dot{x}_{1}^{1 x}, \quad \text { upper MTF (Fig. 2) } \\
c \theta \cdot \dot{x}_{1}^{1 x}-s \theta \cdot \dot{x}_{1}^{1 z}=\dot{x}_{1}^{0 x} \\
s \theta \cdot \dot{x}_{1}^{1 x}+c \theta \cdot \dot{x}_{1}^{1 z}=\dot{x}_{1}^{1 z}
\end{array}\right\} \text { lower MTF (Fig. 2) }
$$

in which $c \theta=\cos (\theta), s \theta=\sin (\theta), \dot{\theta}=\Omega$. 
As in Lagrange's method a set of independent (generalized) velocities is chosen. In this system there are two independent and three dependent velocities (8). It should be noted that although in this case the independent velocities are a subset of the inertial velocities, other than inertial velocities may also be chosen. The velocities of the inertias can be written as a function of the independent velocities by solving (7) for the dependent inertial velocities. The resulting relations will be represented by $T$-matrices (9).

$$
\begin{aligned}
& \text { Independent velocities : } \Omega_{1}^{1 y}, \dot{x}_{1}^{0 z} \\
& \text { dependent velocities: } \dot{x}_{1}^{0 x}, \dot{x}_{1}^{1 x}, \dot{x}_{1}^{1 z} \text {. } \\
& \qquad \dot{\mathbf{q}}_{I}=\mathbf{T}_{I, i} \cdot \dot{\mathbf{q}}_{i},
\end{aligned}
$$

with:

$\dot{\mathbf{q}}_{i}=$ independent velocities,

$\dot{\mathbf{q}}_{I}=$ inertial velocities,

$\mathbf{T}_{I, i}=$ transformation matrix.

In this case the exact form of the matrices is as follows:

$$
\dot{\mathbf{q}}_{I}=\left[\begin{array}{c}
\Omega_{1}^{1 y} \\
\dot{x}_{1}^{0 x} \\
\dot{x}^{0 z}
\end{array}\right], \quad \dot{\mathbf{q}}_{i}=\left[\begin{array}{c}
\Omega_{1}^{1 y} \\
\dot{x}_{1}^{0 z}
\end{array}\right] \quad \text { and } \quad \mathbf{T}_{I, i}=\left[\begin{array}{cc}
1 & 0 \\
\frac{x_{1}^{1 z}}{c \theta} & -\frac{s \theta}{c \theta} \\
0 & 1
\end{array}\right]
$$

The multiport inertia I [Fig. 11(a)] is a $3 \times 3$ matrix, containing all the inertial elements. It will now be transformed to the independent velocities (10). This is equivalent to the transformation of an inertia over an MTF, and results in a virtual inertia $\tilde{I}$ and a gyristor GR [Allen (2)]. The transformation is demonstrated in the partial bond graph of Fig. 11(a) to that of Fig. 11(b). The 1-junction represents the independent velocities.

Multiport inertia : $\quad \mathbf{I}=\left[\begin{array}{ccc}J_{1}^{1 y} & 0 & 0 \\ 0 & m_{1} & 0 \\ 0 & 0 & m_{1}\end{array}\right]$
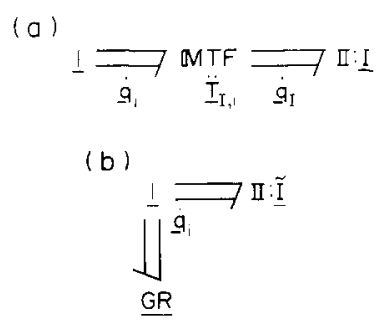

Fig. 11. (a) Bond graph of the junction structure and inertia field. (b) Bond graph with the virtual inertia and gyristor. 
Virtual inertia: $\tilde{\mathbf{I}}=\mathbf{T}_{I, i}^{T} \cdot \mathbf{I} \cdot \mathbf{T}_{I, i}$

$$
=\left[\begin{array}{cc}
J_{1}^{1 y}+\frac{x_{1}^{1 z^{2}}}{c \theta} \cdot m_{1} & -\frac{s \theta}{c \theta} \cdot x_{1}^{1 z} \cdot m_{1} \\
-\frac{s \theta}{c \theta} \cdot x_{1}^{1 z} \cdot m_{1} & \left(1+\left(\frac{s \theta}{c \theta}\right)^{2}\right) \cdot m_{1}
\end{array}\right] .
$$

Gyristor: $\quad \mathbf{G R}=\mathbf{T}_{I, i}^{T} \cdot \mathbf{I} \cdot \dot{\mathbf{T}}_{I, i}$

$$
=\left[\begin{array}{cc}
\frac{s \theta}{c^{3} \theta} \cdot x_{1}^{1 z^{2}} \cdot \Omega_{1}^{1 y} \cdot m_{1}+\frac{x_{1}^{1 z}}{c^{2} \theta} \cdot \dot{x}_{1}^{1 z} \cdot m_{1} & -\frac{x_{1}^{1 z}}{c^{3} \theta} \cdot \Omega_{1}^{1 y} \cdot m_{1} \\
-\frac{s^{2} \theta}{c^{3} \theta} \cdot x_{1}^{1 z} \cdot \Omega_{1}^{1 y} \cdot m_{1}-\frac{s \theta}{c^{2} \theta} \cdot \dot{x}_{1}^{1 z} \cdot m_{1} & -\frac{s \theta}{c^{3} \theta} \cdot \Omega_{1}^{1 y} \cdot m_{1}
\end{array}\right] .
$$

Both I and GR are modulated multiport elements.

Finally the R-, C- and S-elements are defined as multiports too. These elements will also be related to the chosen independent velocities. For all of them transformation matrices $T$ are derived in an equivalent way as for the inertial velocities (11).

$$
\dot{\mathbf{q}}_{\mathrm{C}}=\mathbf{T}_{\mathrm{C}, i} \cdot \dot{\mathbf{q}}_{i}, \quad \dot{\mathbf{q}}_{\mathrm{R}}=\mathbf{T}_{\mathrm{R}, i} \cdot \dot{\mathbf{q}}_{i}, \quad \dot{\mathbf{q}}_{\mathrm{Se}}=\mathbf{T}_{\mathrm{Se}, i} \cdot \dot{\mathbf{q}}_{i}
$$

In which $\dot{\mathbf{q}}_{\mathrm{C}}, \dot{\mathbf{q}}_{\mathrm{R}}, \dot{\mathbf{q}}_{\mathrm{Se}}, \mathbf{T}_{\mathrm{C}, i}, \mathbf{T}_{\mathrm{R}, i}, \mathbf{T}_{\mathrm{Se}, i}$ are defined as:

$$
\begin{aligned}
\dot{\mathbf{q}}_{\mathrm{C}} & =\dot{\mathbf{q}}_{\mathrm{R}}=\left[\dot{x}_{1}^{1 z}\right], \quad \dot{\mathbf{q}}_{\mathrm{Se}}=\left[\dot{x}_{1}^{0 z}\right], \\
\mathbf{T}_{\mathrm{C}, i}=\mathbf{T}_{\mathrm{R}, i} & =\left[\begin{array}{ll}
-\frac{s \theta}{c \theta} \cdot x_{1}^{1 z} & \frac{1}{c \theta}
\end{array}\right], \quad \mathbf{T}_{\mathrm{Sc}, i}=\left[\begin{array}{ll}
0 & 1
\end{array}\right] .
\end{aligned}
$$

The above derived equations can be represented in a multibond graph of the system: the bond graph of Fig. 11 is augmented in Fig. 12 by adding the multiport R-, C-and S-elements and the corresponding transformations (11). A signal vector, drawn with a full arrow, is added to represent dependent velocities. They are, together with the corresponding displacements, required as signals for the modulated transformers and gyristor. Adding causal strokes to this bond graph will now show integral causality for the I- and C-elements. The differential equations can be obtained from this bond graph in an equivalent manner as for single bond graphs. In Eq. (12) these

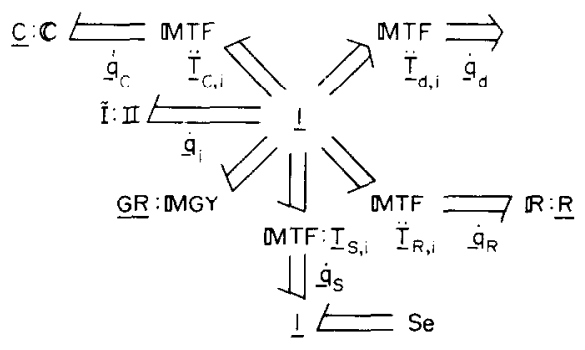

FIG. 12. Multibond graph of the system. 
differential equations are combined in one matrix relation

$$
\mathbf{A}\left[\begin{array}{c}
\ddot{\mathbf{q}}_{i} \\
\dot{\mathbf{q}}_{\mathrm{C}} \\
\dot{\mathbf{q}}_{d}
\end{array}\right]=\left[\begin{array}{ccc}
-\tilde{\mathbf{I}}^{-1} \cdot \mathbf{E} & -\tilde{\mathbf{I}}^{-1} \cdot \mathbf{T}_{\mathrm{C}, i}^{T} \cdot \mathbf{C}^{-1} & \mathbf{0} \\
\mathbf{T}_{\mathrm{C}, i}^{T} & \mathbf{0} & \mathbf{0} \\
\mathbf{T}_{d, i} & \mathbf{0} & \mathbf{0}
\end{array}\right] \cdot\left[\begin{array}{c}
\dot{\mathbf{q}}_{i} \\
\dot{\mathbf{q}}_{\mathrm{C}} \\
\dot{\mathbf{q}}_{d}
\end{array}\right]+\left[\begin{array}{c}
\tilde{\mathbf{I}}^{-1} \cdot \mathbf{T}_{\mathrm{Se}, i}^{T} \\
\mathbf{0} \\
\mathbf{0}
\end{array}\right] \cdot \mathbf{e}_{\mathrm{Se}}
$$

in which

$$
\mathbf{E}=\mathbf{T}_{\mathbf{R}, i}^{T} \cdot \mathbf{R} \cdot \mathbf{T}_{\mathbf{R}, i}+\mathbf{T}_{\mathrm{G}, i}^{T} \cdot \mathbf{G} \cdot \mathbf{T}_{\mathrm{G}, i}^{T} .
$$

An equation has been added to obtain the required dependent velocities $\dot{\mathbf{q}}_{d}$ and their corresponding displacements. In the example the angular displacement $\theta$ of the rod, is required for the transformations $T$.

In the described procedure three main steps from mechanical bond graph to an explicit differential equation are required. Firstly, the derivation of the set of linear equations from the junction structure of the bond graph. Secondly, the solution of these equations to obtain the $T$-matrices. Thirdly the differential equation is constructed using the $T$-matrices and multiport elements. The method described differs from Allen's method: he derived the $T$-matrices by differentiating the displacement equations obtained from the geometry of the mechanical system.

\section{The use of formula manipulation programs}

A general program for formula manipulation, being an interactive program written in Lisp, is Reduce [Hcarn (10)]. With this program onc can manipulate equations as well as large matrices, except for solving linear equations. A collection of procedures and algorithms called Netform [Smit et al. (11)] may be attached to Reduce. These routines are especially made for circuit analysis, including the solution of linear equations. The routines in Netform are very efficient when applied to electrical circuits, because they make use of sparse matrices. Although sparsity is not always a property of the matrices for mechanical systems, the routines are used successfully.

In our application the use of Reduce and Netform is as follows. The velocity relations (7) are given to the program (with the function EQU), as well as a list of unknown variables denoted to the program with the function VAR, see Eq. (13). The variable names in (13) have a one to one relation to those used in Eq. (7). The independent velocities and the sines and cosines are non-constant coefficients. To obtain the matrix relating the inertial velocities to the independent velocities, the inertial velocities are solved. From this the corresponding $T$-matrix is known. The same technique may be used to obtain all the $T$-matrices. After the multiport elements have been given to the program, the differential equation is obtained in formula form and can be written as a file in Fortran format. The differential equation may be integrated with a standard simulation program, which allows input in equation form. Being familiar with the very user friendly commands in TUTSIM [Meerman (12)], a program was written with mainly the same commands, but a model description in formula form. This program, called MBGSIM, has been used 
to simulate the industrial manipulator [Bos (13)].

$$
\begin{aligned}
& \mathrm{EQU}-x 1 z 2 * \text { omega } 1 y 1 \quad-v 1 x 1, \\
& \cos (\text { teta }) * v 1 \times 1-\sin (\text { teta }) * v 1 z 1-v 0 \times 1 \text {, } \\
& \sin (\text { teta }) * v 1 \times 1+\cos (\text { teta }) * v 1 z 1-v 0 z 1 \text {; }
\end{aligned}
$$

VAR $v 1 \times 1, v 1 z 1, v 0 \times 1$;

in which:

$$
\text { viap }=\dot{\mathbf{x}}_{p}^{i a}, \quad a=x, y, z .
$$

Due to the use of the formula manipulation programs it is easy to experiment with the equations. So it was found that a relation exists between the choice of the generalized velocities and the complexity (in the number of algebraic operations) of the differential equation.

The best results were found when the generalized velocities are angular velocities of bodies in body coordinates and linear velocities of masses in inertial coordinates. A bad choice results in very large $T$-matrices; a good choice in simple matrices in which it is possible to check the equations by comparing the elements in the $T$-matrices with the junction structure of the bond graph or with the original system.

As described, the formula manipulation program has been used to solve equations, to obtain the $T$-matrices. This solution, however, requires an excessive amount of core (roughly 0.25-1 Mbyte for a system like the industrial manipulator). A good choice of the independent velocities decreases the core demand considerably. To make quantitative statements more study is required.

\section{Simulation of a robot}

The above described method is used to model and simulate an industrial manipulator of which the model has already been described. The three steps to obtain the differential equation will not be shown in detail but will be discussed briefly.

$$
\dot{\mathbf{q}}_{\mathrm{C}}=\left[\begin{array}{c}
\dot{x}_{\mathrm{C} 2} \\
\dot{x}_{\mathrm{C} 3}
\end{array}\right], \quad \dot{\mathbf{q}}_{i}=\left[\begin{array}{c}
\Omega_{2,1}^{1 x} \\
\Omega_{1}^{1 z} \\
\dot{x}_{30}^{2 y, 2}
\end{array}\right], \quad \dot{\mathbf{q}}_{d}=\left[\begin{array}{c}
\Omega_{2,1}^{1 x} \\
\Omega_{1}^{1 z} \\
\dot{x}_{35}^{0 x} \\
\dot{x}_{35}^{0 x} \\
\dot{x}_{35}^{0 z}
\end{array}\right], \quad \dot{\mathbf{q}}_{\mathrm{R}}=\left[\begin{array}{c}
I_{1} \\
\Omega_{2,1}^{1 x} \\
\dot{x}_{3,2}^{2 y}
\end{array}\right], \quad \mathbf{S}=\left[\begin{array}{c}
S_{\mathrm{e} 1} \\
S_{\mathrm{f} 2} \\
S_{\mathrm{f} 3}
\end{array}\right] .
$$

The velocity relations (7) are derived from the junction structure of the bond graph. By choosing the independent velocities and denoting the velocities of the elements (14), the calculation of the $T$-matrices can be done. After adding the element fields the differential equation may be constructed in a Fortran subroutine.

Some simulation results are shown in Fig. 13. This figure shows the high frequencies, due to oil compressibility. 


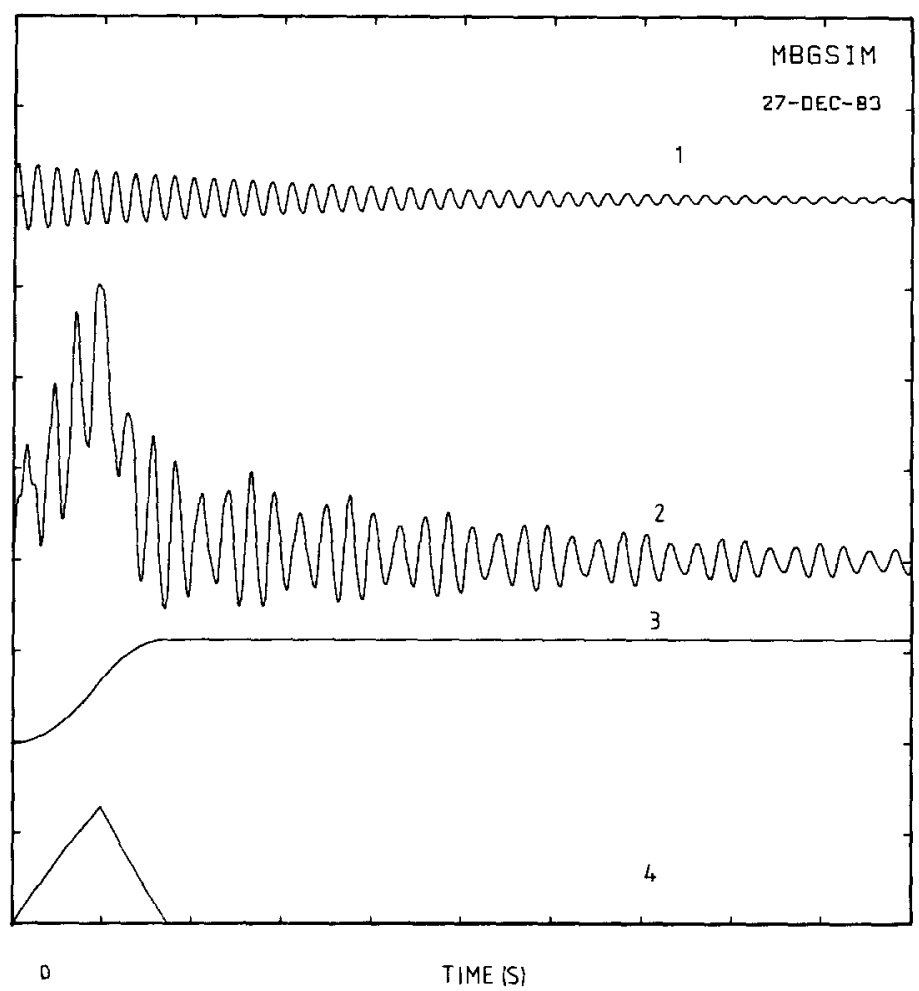

FIG. 13. Response to a positive pulse of the electric motor on the vertical axis of body 1 , followed by an equal negative pulse. Curve 1 represents the angle, between the $y$-axes of the bodies 2 and 3 with the line which has an angle of $0.25 \mathrm{rad}$ with the horizontal (the starting position); curve 2 represents the displacement in the $y 2$ direction of body 3 with respect to body 2 ; curve 3 is the angular displacement of the robot on the vertical axes; curve 4 is the vertical angular velocity.

\section{Summary}

The presented method of modelling 3-D mechanical systems with bond graphs has been formalized by:

(i) the introduction of an elaborate notation of velocities,

(ii) the introduction of a general bond graph describing a body in a multibody system,

(iii) a word bond graph in relation with the description of multibody systems, in which each block represents a link in a multibody system. Each block (link) can be worked out in detail in a standard way.

A method is described to solve the causality problem related to the existence of both integral and differential causality in a mechanical bond graph. This method transforms the mechanical bond graph to a multibond graph with integral causality only. This multiport bond graph is equivalent to an explicit differential equation 
which can be solved by a simulation program. Programs for formula manipulation are used to transform the mechanical bond graph to the multibond graph. Due to this formula manipulation it becamc possiblc to expcriment with the choice of independent (generalized) velocities and obtain efficient results. The core demand of the formula manipulation is excessively large and will be investigated in the near future.

\section{References}

(1) J. Wittenburg, "Dynamics of Systems of Rigid Bodies", B. G. Teubner, Stuttgart, 1977.

(2) R. R. Allen, "Dynamics of mechanisms and machine systems in accelerating reference frames", Trans. ASME J. Dynamic Syst. Measure. Control, Vol. 103, pp. 395-403, Dec. 1981.

(3) M. J. L. Tiernego and J. J. van Dixhoorn, "Bond graph modelling and simulation techniques applied to a three axes driven pendulum", Int. J. Modelling Simulation, Vol. 1, No. 1, pp. 62-65, 1981.

(4) M. J. L. Tiernego and A. M. Bos, "Modelling the dynamics and kinematics of mechanical systems with multibond graphs", J. Franklin Inst., Vol. 319, No. 1/2, pp. 37-50, 1985.

(5) P. C. Breedveld, "Proposition for an unambiguous vector bond graph notation", Trans. ASME J. Dynamic Syst. Measure. Control, Vol. 104, No. 3, pp. 267-270, Sept. 1982.

(6) D. L. Margolis and D. C. Karnopp, "Bond graphs for flexible multibody systems", Trans. ASME J. Dynamic Syst. Measure. Control, Vol. 101, pp. 50-77, March 1977.

(7) R. R. Allen, "Multiport representation of inertia properties of kinematic mechanisms", $J$. Franklin Inst., Vol. 308, No. 3, pp. 235-253, Sept. 1979.

(8) J. J. van Dixhoorn, "Simulation of bond graphs on mini-computers", Trans. ASME J. Dynamic Syst. Measure. Control, Vol. 99, pp. 9-14, March 1977.

(9) R. R. Allen and S. Dubowsky, "Mechanisms as components of dynamic systems: A bond graph approach", Trans. ASME J. Engng Ind., Vol. 99, No. 1, pp. 104-111, Feb. 1977.

(10) A. C. Hearn, "Reduce 2 users manual", University of Utah, 1973.

(11) J. Smit, J. A. Hulzen and B. J. A. Hulshof, "Netform and code optimizer manual", $A C M$ Sigsam Bull., Vol. 15, No. 4, pp. 23-32, Nov. 1981.

(12) J. W. Meerman, "Bond graph modelling and simulation techniques; software for the simulation of continuous dynamic systems", Int. J. Modelling Simulation, Vol. 1, No. 1, pp. 52-56, 1981.

(13) A. M. Bos, "MBGSIM users manual", No. 1241.3442, Twente University of Technology, Department of Electrical Engineering, Enschede, The Netherlands (in Dutch), 1983. 